EPJ Web of Conferences 16, 03002 (2011)

DOI: $10.1051 /$ epjconf/20111603002

(C) Owned by the authors, published by EDP Sciences, 2011

\title{
Sub-Airy disk angular resolution with high dynamic range in the near-infrared
}

\author{
A. Richichi \\ European Southern Observatory, Karl-Schwarzschildstr. 2, 85748 Garching, Germany
}

\begin{abstract}
Lunar occultations (LO) are a simple and effective high angular resolution method, with minimum requirements in instrumentation and telescope time. They rely on the analysis of the diffraction fringes created by the lunar limb. The diffraction phenomen occurs in space, and as a result LO are highly insensitive to most of the degrading effects that limit the performance of traditional single telescope and long-baseline interferometric techniques used for direct detection of faint, close companions to bright stars. We present very recent results obtained with the technique of lunar occultations in the near-IR, showing the detection of companions with very high dynamic range as close as few milliarcseconds to the primary star. We discuss the potential improvements that could be made, to increase further the current performance. Of course, LO are fixed-time events applicable only to sources which happen to lie on the Moon's apparent orbit. However, with the continuously increasing numbers of potential exoplanets and brown dwarfs beign discovered, the frequency of such events is not negligible. I will list some of the most favorable potential LO in the near future, to be observed from major observatories.
\end{abstract}

\section{THE METHOD}

The geometry of a lunar occultation (LO) event is sketched in Figure 1. The lunar limb acts as a straight diffracting edge, moving across the source with an angular speed that is the product of the lunar motion vector $\mathrm{V}_{\mathrm{M}}$ and the cosine of the contact angle $\mathrm{CA}$. This produces a scan of the source along an angle which is the algebric sum of the position angle PA and local limb slope $\psi$. Lunar mountains have a limited effect since limb irregularities are averaged over scales much larger than the geometric size of the star at the Moon. The result is a light curve similar to Figure 1, simulated for a reappearance under typical conditions in the near-IR, without noise. Abscissae are marked in seconds from the time of geometrical occultation, and intensity is arbitrary.

The light curve has characteristic fringes of increasing frequency and decreasing amplitude. These fringes embed information on the brightness distribution of the source along the direction of scan, and with proper analysis information can be retrieved on milliarcsecond (mas) scales. The main advantage of the LO method is that observations are very economical in terms of instrumentation and of telescope time. Essentially, fast photometry on ms scales is required, without needs of calibration observations. Data reduction is also not very intensive, compared to other high angular resolution techniques. The downside is of course that targets cannot be chosen at will: the Moon apparent orbit covers only about $10 \%$ of the sky, with a repetition rate of about 18.5 years (Saros cycle). Since LO are fixed time events, bad weather or technical downtimes are especially frustrating.

\section{OBSERVATIONS}

LO events have been observed routinely since the 1970's with fast photometers, but their use has gradually decreased for a number of reasons. The fact that millisecond time resolution capabilities

This is an Open Access article distributed under the terms of the Creative Commons Attribution-Noncommercial License 3.0, which permits unrestricted use, distribution, and reproduction in any noncommercial medium, provided the original work is properly cited. 

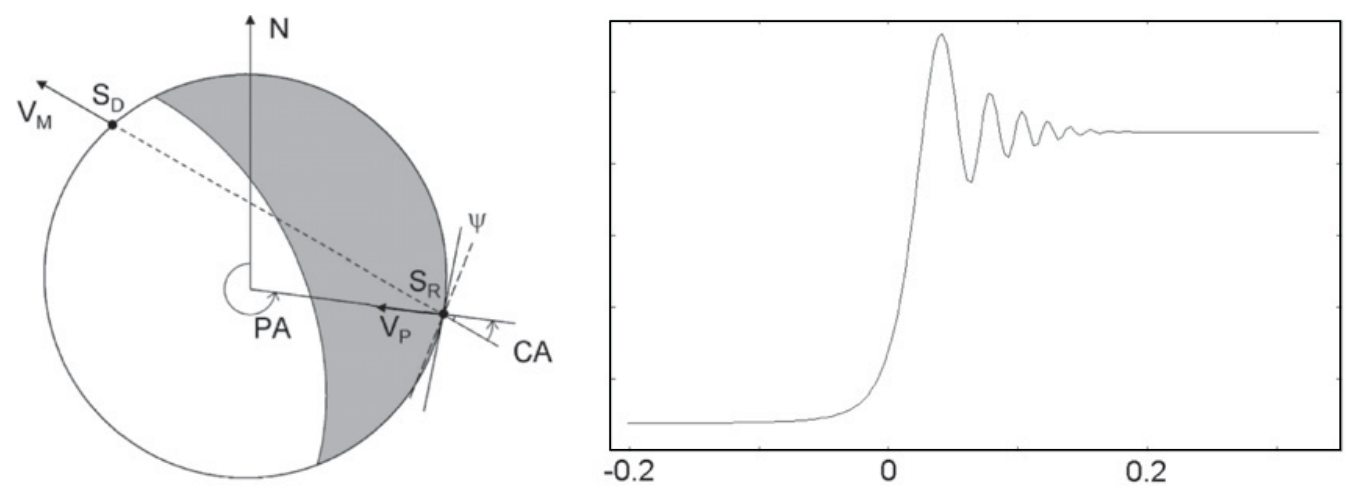

Figure 1. Geometrical elements of an occultation (left), and model of a light curve with relative time in seconds (right). See text for details.
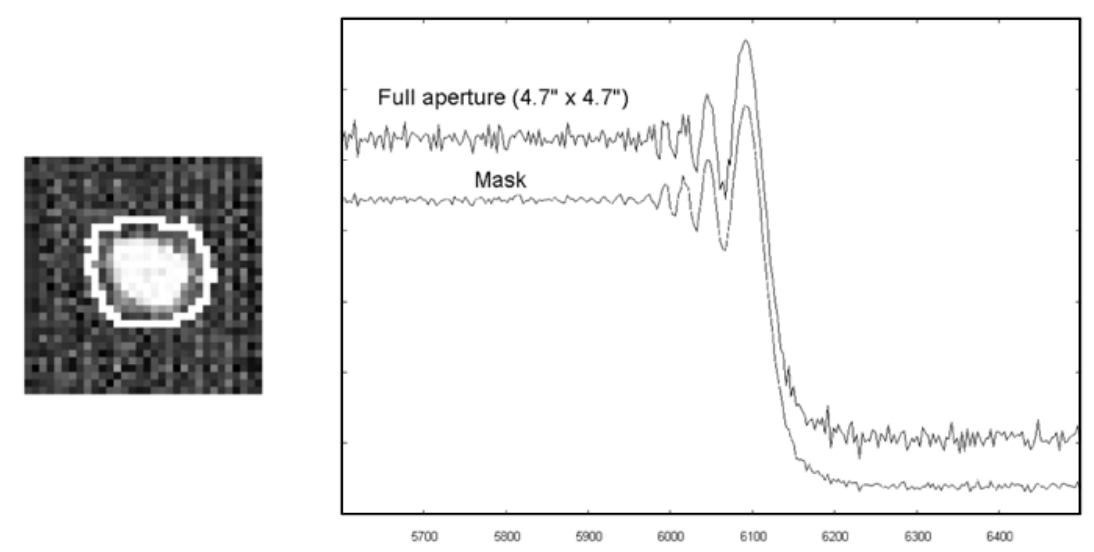

Figure 2. Left: average of 5,000 frames at $3.2 \mathrm{~ms}$ DIT, of a LO event. Right: light curves extracted from the full aperture, and using the mask defined by the sextractor algorithm in our automated pipeline [1], as shown by the solid contour overimposed on the average of the frames.

are not easily found in modern large telescope instruments was certainly a factor. Additionally, IR all-sky surveys had been traditionally limited (and incomplete) to very bright magnitudes, for which LO had already provided almost all possible results. The incresasing availability of longbaseline interferometers, operating with similar sensitivity and angular resolution but with more ease of repeatability, also influenced continuing efforts with LO.

A significant breakthrough has been reached in the recent years, thanks on one side to the availability of deep IR-surveys, and on the other side to the ability to use fast read-out modes on subwindows of the IR detectors commonly found on large telescopes. Given that LO are affected by high background values, the possibility to use array detectors has a great potential of increasing the effective sensitivity.

Figure 2 shows data taken with the ISAAC instrument at the ESO VLT. Two light curves are shown, obtained from the full window as in a photometer, and by using an extraction mask [1]. To make a comparison, in the 1980 's a $4 \mathrm{~m}$ telescope equipped with a fast IR photometer was sensitive to $\mathrm{K} \approx 9$ mag, but predictions could be made only for a small number of events per night, generally one or two dozens in the luckiest situations. At present, a $10 \mathrm{~m}$ class telescope with an array detector is sensitive to $\mathrm{K} \approx 12.5 \mathrm{mag}$, and using the 2MASS Catalogue predictions run in the order of hundreds of events on each night, up to tens fo thousands in special cases (see Figure 3). Of course, due to telescope overheads 


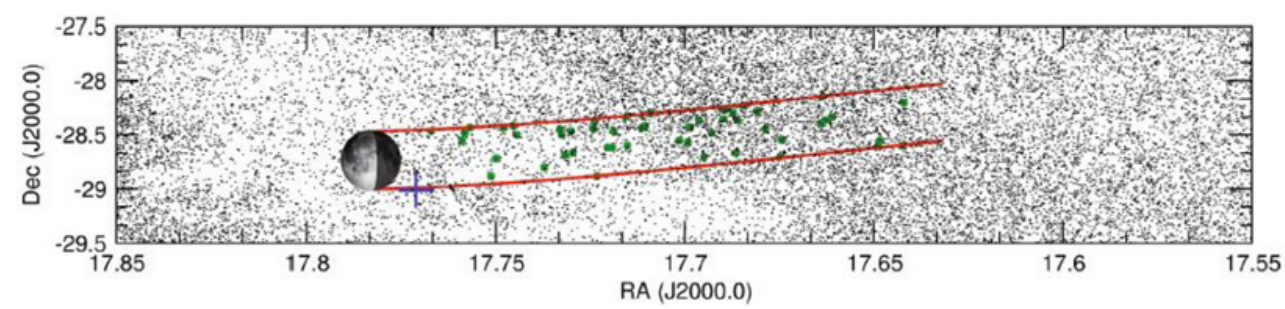

Figure 3. Location of sources occulted by the Moon in the night of March 21, 2006 (reappearances). The small dots represent sources in the 2MASS catalog with magnitude $\mathrm{K} \leq 7.5$. The heavier dots are the sources for which we could record a lunar occultation. The apparent path of the Moon, moving from West to East, is also shown. The cross marks the Galactic Center. See [2].
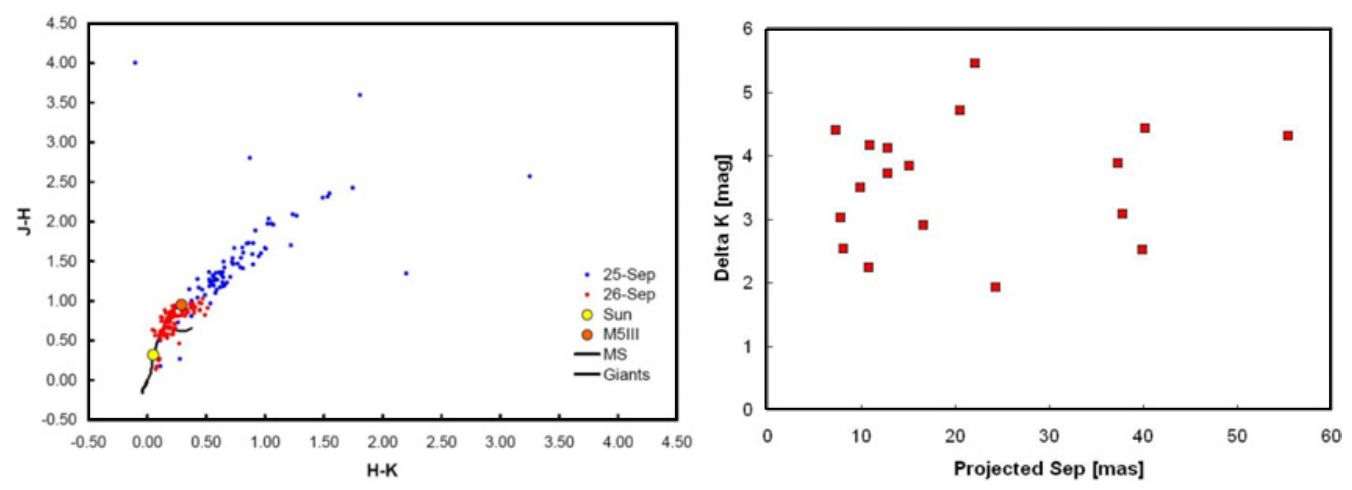

Figure 4. Left, color-color location of the sources described in the text. The extreme reddening can be appreciated (compare with positions of main sequence, giants, and the Sun). Right, parameters of the binaries detected in this sample.

it is not possible to observe all of them, but recent observations by our group have collected up to 100 events in one half night, or an average of one every three minutes including overheads for pointing and data storage.

An additional advantage of telescopes in the $8-10 \mathrm{~m}$ class is that scintillation, a major source of noise for bright sources, is drastically reduced with respect to smaller telescopes. This provides excellent SNR, which in turn leads to very precise measurements: our observations in a broad-band $\mathrm{K}$ filter with the ISAAC instrument at the VLT reach SNR>200 for bright sources, and have a limiting angular resolution below 1 mas.

Since the start of our observations at the VLT three years ago, a total of about 500 events have been recorded, with magnitudes as faint as $\mathrm{K} \sim 11$. An accurate census of the results for the latest observations, completed in September 2009, is still in progress but we can already anticipate that the total number of binaries detected is $\sim 50$, the large majority of which not previously known and in fact without known optical counterparts.

Figure 4 shows the colors of the sources detected during two half-nights in September 2009. The extreme reddening can be appreciated, mainly due to interstellar extinction in the general direction to the Galactic Center. However, cases of extreme local colors can also be seen. Only very few of the sources have known optical counterparts. In this sample of $\sim 180$ sources, a preliminary analysis shows that 18 sources have close companions (some of them are actually triple, but we only refer to the brightest two components here). In Figure 4, it can be seen that the magnitude differences are quite high, making the follow up of these sources quite challenging by any other method. In fact, the pairs with projected 

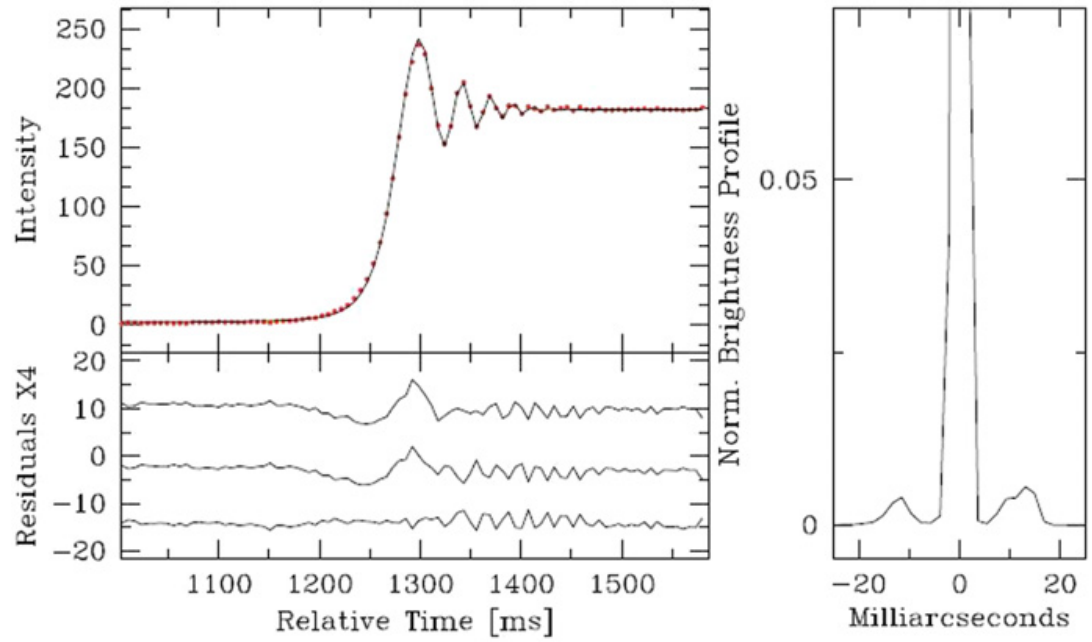

Figure 5. Left: upper panel, data and best fit for 2MASS 17453224-2833429, a SiO maser with no optical counterpart. The lower panel shows on an enlarged scale the residuals of three different fits, see [2] for details. Right: brightness profile reconstructed by a model-independent method, with the typical signature of a circumstellar shell.

separations $<20$ mas (i.e. about $1 / 3$ of the Airy disk of a large telescope at this wavelength) would require long-baseline interferometers, which however in most cases are unable to detect these systems due to their faintness.

In addition to binary stars, LO are also ideal to study features and structures with low contrast, well inside the Airy disk of the telescope. An example is given in Figure 5, which shows the detection of an IR circumstellar shell associated with a radio maser. Details about the size and the distance are given in [2]. Here we mention that this and other measurements have proven the capability of the LO technique at a large telescope to achieve a dynamic range of 7-8 magnitudes in broad $\mathrm{K}$ band, on angular scales which are a fraction of the Airy disk.

\section{References}

[1] O. Fors, A. Richichi, X. Otazu, J. Nuñez, A\&A, 480, 297 (2008)

[2] A. Richichi, O. Fors, E. Mason, J. Stegmeier, T. Chandrasekhar, A\&A, 489, 1399 (2008) 